\title{
DÉCISION ADMINISTRATIVE ET DÉMOCRATIE ADMINISTRATIVE
}

UDK: 342.9

Primljeno: 1. rujna 2018.

Izvorni znanstveni rad

La démocratie administrative n'est pas un mécanisme inutile. Par conséquent, les éléments suivants sont considérés dans cet article: référendum sur l'initiative citoyenne au niveau local, organes consultatifs au niveau local qui rassemblent les fonctionnaires au niveau local et les clients finaux, les messages issus de la démocratie administrative, etc. Il souligne que la démocratie administrative favorise la participation dans la prise de décision publique par divers professionnels et la nécessité de la présence systématique de divers participants à la discussion (associations civiles, etc.). Enfin, les inconvénients de la démocratie administrative sont également observés.

\section{Mots wclés: administration, décisions, démocratie administrative}

Je voudrais tout d'abord exprimer mes plus vifs remerciements aux organisateurs de ces rencontres, notamment M. Marc Gjidara, de m'avoir convié à ces rencontres juridiques et administratives franco-croates de droit. Je voudrais ensuite préciser à quel point, sur ce thème de l'Etat que nous évoquons pendant ces deux jours, il m'a semblé important de vous apporter l'éclairage du droit français sur les rapports qu'entretiennent les notions de décision administrative et de démocratie administrative.

La première n'a pas besoin d'être longuement rappelée. Chacun le sait, elle est l'instrument de prédilection de la réalisation par l'Administration de ses missions : l'Administration agit, exécute la loi, maintient l'ordre public, assure des prestations de services au moyen de l'édiction de décisions. Et en France, pays dans lequel le droit administratif a été entièrement forgé autour de la décision administrative, son édiction tout autant que son contrôle, la décision administrative, c'est tout acte juridique unilatéral, qu'il soit individuel ou règlementaire, édicté par les autorités administratives ou par les personnes privées associées à l'action publique. Le droit administratif français est avant tout un droit de la décision, laquelle incarne ses valeurs, ses principes généraux, puisque c'est l'instrument du triomphe de l'intérêt général sur les égoïsmes privés et les intérêts particuliers, et c'est aussi l'objet du contrôle du juge administratif, chargé de vérifier que, en décidant, l'Administration a bien respecté la loi, expression de la volonté générale.

Par contraste, le concept de démocratie administrative est étranger aux préoccupations traditionnelles du droit administratif français : il est plus familier 
des droits étrangers, anglo-saxons notamment, mais aussi des régimes juridiques applicables dans les pays qui ont accédé plus récemment à la démocratie libérale, comme l'Espagne, le Portugal ou les anciens satellites de l'ex-Union soviétique. Il est en effet intéressant de souligner que la démocratie administrative est une préoccupation beaucoup plus développée dans les pays qui ont été longtemps été privés de démocratie politique. En France du reste, quand le droit administratif s'est préoccupé de proclamer quelques garanties, comme le respect par l'Administration des droits de la défense, comme l'obligation d'entendre préalablement tout administré allant faire l'objet d'une mesure défavorable, c'est parce que la France sortait des heures sombres du gouvernement de Vichy et de la seconde guerre mondiale. Ce qui montre à quel point les concepts de démocratie politique et de démocratie administrative entretiennent de forts liens de parenté. En réalité, pour faire simple, la démocratie administrative est un concept juridique militant qui reprend à son compte les deux grands idéaux de la démocratie politique : d'une part, la transparence, en ce sens que l'Administration doit être ouverte sur l'extérieur pour que les administrés puissent exercer leur droit de contrôle sur leurs agents, comme la citoyenneté politique leur permet de contrôler leurs représentants ; d'autre part, la participation, en ce que le propre de la citoyenneté est de pouvoir prendre part à la vie publique.

D'origine étrangère, le concept de démocratie administrative n'en exerce pas moins une influence sur le droit administratif français contemporain, qui, sous l'effet du droit de l'Union européenne, en est venu à reprendre à son compte les concepts de démocratie administrative, de citoyenneté administrative, de droits des administrés à la transparence et à la participation. Mais ce qui est intéressant à noter, c'est que, précisément, le cas français, par sa conversion récente à la démocratie administrative, révèle, dans le cas des décisions administratives, tout autant, les avantages que les inconvénients, les mérites que les limites de la démocratie administrative.

\section{Quelques mots tout d'abord sur les mérites de la démocratisation de la décision administrative.}

Que doit le droit administratif français à l'idéal de démocratie administrative ?

D'abord, une exigence de transparence, qui permet à l'administré d'exiger de l'Administration qu'elle informe, qu'elle s'explique, qu'elle ne se retranche pas derrière le secret et l'opacité dans ses processus décisionnels : reconnaissance en 1978 d'un droit d'accès aux documents détenus par l'Administration ; fin, depuis 2000, de l'anonymat des décisions administratives, avec obligation pour l'auteur d'une décision d'indiquer ses nom, prénom, qualité et des signer l'acte ; politique d'ouverture des données que l'Administration peut prendre en compte pour se décider dans un sens et pas dans un autre, ce que l'on appelle la politique de l'Open et du Big Data promue et règlementée depuis 2016.

Ensuite, la démocratie administrative, c'est la mise en avant d'une exigence de participation qui se manifeste de diverses manières:

- Par un droit d'initiative des administrés, à l'image du référendum 
d'initiative populaire en matière locale ;

- Par l'institution de cercles de personnes intéressées visant à réunir les destinataires d'une norme, à l'image des commissions consultatives des services publics locaux qui réunissent élus et usagers ;

- Par l'institution de consultations fermées, confiées à des organismes consultatifs ;

- Par l'institution, plus récente, de consultations ouvertes sur internet, aussi bien pour permettre au ministère de l'économie d'organiser de vastes consultations sur les projets de textes relatifs à la commande publique que pour permettre à une région de consulter la population sur un changement de nom, comme l'a montré un récent arrêt du Conseil d'Etat de 2017, consultations ouvertes qui doivent désormais se substituer aux commissions consultatives lorsqu'elles portent sur le même sujet ;

- Par des procédures d'enquête publique et de débat public, qui, à compter de 1983 et de 1995, se sont d'abord développées en matière d'aménagement (expropriation, urbanisme) et se sont ensuite étendues à la matière environnementale au point que, en droit français, sous l'effet de la convention d'Aarhus, le principe de participation en matière environnementale a même acquis valeur constitutionnelle, via l'article 7 de la Charte de l'environnement, complété par de nombreuses lois, en 2012 et 2016, qui aménagent avec détails les modalités de cette participation du public affecté par l'impact d'une décision administrative sur leur environnement naturel ou urbain.

Quels enseignements positifs peut-on tirer de cette promotion de la démocratie administrative ? J'en vois deux trois principaux.

Tout d'abord, cet avènement de la démocratie administrative permet au droit français de percevoir différemment l'intérêt général. Il ne s'agit pas ici de tout remettre en cause et de dire qu'il est bon que le droit français se convertisse à la définition anglo-saxonne d'un intérêt général conçu comme la somme des intérêts particuliers entre lesquels l'Administration ne devrait qu'arbitrer. La conception continentale et romaine de l'utilitas publica, d'un intérêt général distinct et supérieur aux intérêts particuliers, conserve toute son influence. Mais à force de voir dans l'intérêt général un intérêt transcendantal définie exclusivement par une élite administrative détentrice du savoir de ce qui est bon pour le bien commun, on finit trop en France par voir dans l'intérêt général une donnée immanente et nécessairement bonne en elle-même pour l'utilité de tous. Le principe de participation a au moins le mérite de faire redescendre l'intérêt général du ciel vers la terre, de faire prendre conscience qu'il est l'expression d'un choix politique et que, dans la détermination de ce choix, il entre, sinon une part de compromis, du moins une prise en compte des intérêts contradictoires, ce qui implique d'écouter et de récolter la diversité des points de vue qui peuvent s'exprimer dans la communauté des administrés. 
Ensuite, la promotion de la démocratie administrative a le grand mérite d'arracher le droit administratif français à son prisme contentieux. Ici aussi, il ne s'agit pas de tout remettre en cause. Le passé de la France explique pourquoi son droit administratif privilégie le contrôle du juge et les garanties contentieuses. Dans un pays qui a identifié l'Administration au pouvoir exécutif et l'a défini comme la fonction d'exécution des lois, il n'était pas absurde d'affirmer que l'Administration doit obéissance à la loi et qu'il revint au juge de la censurer toutes les fois mais uniquement ces fois-là - où elle agirait contra legem. Mais le problème est que, dans les domaines comme l'urbanisme ou l'environnement où les autorités administratives disposent nécessairement d'un large pouvoir d'opportunité ou d'un pouvoir discrétionnaire pour suggérer seulement des comportements ou agir au plus près des faits concrets, un pur contrôle juridictionnel de légalité rencontre nécessairement des limites, et bute précisément sur l'interdiction pour le juge de s'immiscer dans l'appréciation des choix purement opportuns de l'Administration. Il est donc salutaire que le droit puisse, en amont du processus décisionnel, contraindre l'administration à entendre tous les points de vue, à savoir ce que les destinataires d'une future décision administrative, ce que les intéressés, pensent du projet d'aménagement de l'Administration. En écoutant, en consultant, en faisant la synthèse des points de vue exprimés, en confrontant ses arguments à ceux des administrés, l'Administration est mieux éclairée sur la réelle opportunité de ses choix, de ce qu'elle entend mettre en œuvre.

Enfin, dans un droit administratif français qui a longtemps été influencé par la vision de l'école objectiviste, de Léon Duguit et de ses disciples, la démocratie administrative introduit une dose de subjectivité dans un droit administratif profondément objectif, centré sur l'objet de l'action administrative et non sur ses sujets. D'une part, dans la mesure où elle permet la prise en compte préalable du point de vue des particuliers, la démocratie administrative participative assure la concrétisation et l'effectivité des droits subjectifs des particuliers mis en jeu par le projet de décision de l'Administration. D'autre part, sans exagérer le constat et faire croire que le droit administratif serait devenu l'égal du droit allemand par exemple, l'idéal de démocratie administrative, en assurant la concrétisation d'un droit de communication, d'un droit à l'information, d'un droit à la motivation, d'un droit à la participation, participe d'un meilleur ancrage des droits subjectifs des administrés, conception qui a longtemps été écarté par le droit français.

Tous ces mérites ne doivent pas occulter les défauts de cette démocratisation de la décision administrative. Le droit français, qui ne s'est converti que récemment à ce nouveau dogme, permet de prendre un peu de distance avec ce phénomène et d'en mieux percevoir les limites.

C'est le second point que je voudrais examiner.

Parce que le droit administratif s'est un peu moins que d'autres enthousiasmé pour la démocratie administrative, il permet de prendre la mesure des réserves qu'implique cet idéal, du moins pour ce qui concerne l'édiction des décisions de 
l'Administration. Dans bien des cas, la démocratie administrative s'avère un outil inutile et un concept détourné.

Un outil inutile tout d'abord. D'un point de vue quantitatif, la participation demeure numériquement faible : les référendums d'initiative populaire en matière locale ne sont quasiment jamais initiés ; les débats publics n'attirent pas les foules ; les consultations ouvertes et autres enquêtes publiques n'attirent pas beaucoup de monde, ce qui contraste avec l'importance des projets envisagés et l'ampleur des informations diffusées. Il résulte un problème de légitimité, puisque la représentativité des participants apparaît comme très discutable pour légitimer la décision publique finale. Mais, le problème n'est pas purement quantitatif. Dans une très intéressante étude de John Hibbing et Elisabeth Thiess-Moss (Stealth democracy, 2002), des chercheurs ont pu montrer que les citoyens américains avaient un désintérêt qualitatif pour les procédures de participation, qu'ils n'avaient en fin de compte aucun désir de s'impliquer dans la gestion administrative et la prise de décision publique, car ils avaient une préférence pour le système classique d'élus et d'agents responsables leur permettant de rester en retrait de la gestion, même à leur niveau, de l'appareil administratif de l'Etat. Ce désintérêt quantitatif et qualitatif met à mal le concept de démocratie administrative, qui repose sur l'affirmation, la croyance, d'une volonté de réappropriation citoyenne de la res publica.

La démocratie administrative serait également inutile car inefficace. D'une part, la technicité des sujets d'aménagement sur lesquels on consulte des administrés, l'ampleur des informations qu'on leur délivre, l'absence des codes de communication propres à la bureaucratie font que les participants à une procédure administrative consultative n'ont pas nécessairement les codes de compréhension et d'argumentation propres à la pratique administrative. D'autre part surtout, l'efficacité de la démocratie administrative est logiquement tributaire de son impact sur la décision finale de l'Administration, Or, à l'exception peutêtre du référendum local décisionnel, mais si peu utilisé en France depuis 2003, la démocratie administrative n'est pas synonyme de co-décision ; in fine, c'est toujours l'Administration seule qui décide. Dès lors qu'il n'existe pas vraiment d'obligation juridique de prendre en compte les résultats d'une consultation, d'adopter la décision qui serait la synthèse des points de vue exprimés, ou ne serait-ce que de motiver pourquoi la décision finale s'écarte de la consultation, ce qui en France n'a guère de chance d'arriver, l'obligation de motivation ne s'imposant qu'aux décisions administratives individuelles défavorables, la démocratie administrative est destinée à rester largement inefficace et inutile, sans influence sur le sens de la décision prise in fine par l'Administration. L'affaire récente en France du projet de construction d'un aéroport à Notre-Dame-des-Landes, où le résultat d'une consultation populaire favorable à la construction est resté sans effet sur la décision finale de renoncer à sa construction, en offre une illustration exemplaire.

Ce constat conduit à considérer que la démocratie administrative n'est pas seulement un outil inutile. C'est aussi un concept détourné. Détournement de la part de l'Administration qui, sachant qu'une procédure consultative a été organisée 
sans avoir pour autant d'effet sur le contenu de la décision finale, en fait un outil de légitimation de ses décisions : alors même que le résultat final s'en est détaché, l'Administration peut toutefois faire valoir qu'elle a sollicité l'avis des administrés eux-mêmes et diffuser ainsi dans la société tout entière la croyance illusoire en la légitimité systématique et a priori des décisions publiques qui, si elles restent juridiquement unilatérales, ne seraient jamais prises sans et contre ceux auxquels elles sont destinées. N'oublions pas que, il y a déjà plus d'un siècle, le grand juriste et vice-président du Conseil d'Etat Édouard Laferrière observait que les formalités imposées à l'Administration ne l'étaient que "dans l'intérêt de l'acte administratif lui-même, de sa correction, de sa maturité, en un mot dans un but de bonne administration ». Où l'on voit que le concept de bonne administration n'a rien de nouveau et ne signifie pas nécessairement ce que l'on croit.

Détournement donc par l'Administration, mais aussi par certains groupes d'administrés. La théorie américaine de la capture, popularisée par George Stigler, trouve une nouvelle illustration en matière de démocratie administrative. Parce que la participation reste faible, parce que les consultations ouvertes sur internet ne saurait faire oublier l'existence d'une fracture numérique qui exclut toute une partie des administrés de la démocratie administrative, parce que la technicité des sujets exclut de fait les administrés sans expérience, la démocratie administrative favorise la participation à la prise de décision publique « de ceux qui disposent d'un savoir expert ; « la présence systématique aux débats d'acteurs (associations, collectifs, etc.), ayant acquis une maîtrise des démarches consultatives par rapport aux individus profanes crée un danger d'instrumentalisation de celles-ci pour la promotion d'intérêts particuliers ou corporatistes ».

On le voit, la démocratie administrative, en matière de décision administrative n'a pas que des vertus. Pour conclure, je dirais plutôt qu'elle ni bonne ni mauvaise en soi. Tout dépend du contexte de l'ordre juridique dans lequel on souhaite l'acclimater. Soit un ordre juridique, en matière administrative, accorde depuis longtemps une large place à la collaboration de l'Administration avec les administrés, auquel cas rien ne s'oppose à une promotion des modalités de la démocratie administrative. Soit un ordre juridique, comme celui de la France, reste attaché à l'unilatéralité de l'action administrative, auquel cas le développement de la procédure administrative non contentieuse ne peut pas devenir le seul remède à l'arbitraire administratif, et il convient de conserver au contrôle en aval du juge administratif un rôle de premier plan dans la lutte contre les irrégularités et les dysfonctionnements de l'Administration. C'est pourquoi je trouve dangereux la politique actuelle du Conseil d'Etat, qui par des arrêts comme Danthony en 2011 et le tout récent arrêt CFDT Finances du 18 mai 2018, réduit la portée des vices de procédure et de forme dans le contentieux de l'annulation des décisions de l'Administration. On ne peut vouloir en même temps prétendre favoriser la promotion de la démocratie administrative en France et considérer en même temps que, au stade ultérieur du contentieux, les irrégularités formelles affectant les procédures participatives n'entachent pas d'illégalité les décisions administratives auxquelles elles ont abouti. Dans un Etat qui peut légitimement vouloir préférer la procédure administrative contentieuse à la 
procédure administrative non contentieuse, on ne peut raisonnablement restreindre la première alors que la seconde ne la remplacera jamais.

\section{ADMINISTRATIVE DECISIONS AND ADMINISTRATIVE DEMOCRACY}

Administrative democracy is not a useless mechanism. Therefore, the following is considered in this article: referendum upon citizen initiative at local level, advisory bodies at local level which gather officials at local level and ultimate customers, the messages which emerge from administrative democracy, etc. It stresses that administrative democracy favors participation in public decision making by various professional people, and the need for the systematic presence of various participants in discussion (civil associations, etc.). Finally, the downsides of administrative democracy are also observed.

Key words: administration, decisions, administrative democracy

\section{UPRAVNA ODLUKA I UPRAVNA DEMOKRACIJA}

Administrativna demokracija nije beskoristan mehanizam. Stoga se u ovom članku razmatra: referendum o građanskoj inicijativi na lokalnoj razini, savjetodavna tijela na lokalnoj razini koja okupljaju dužnosnike na lokalnoj razini i krajnje korisnike, poruke koje proizlaze iz upravne demokracije itd. Naglašava da administrativna demokracija favorizira sudjelovanje u javnom odlučivanju raznih stručnih ljudi i potrebi sustavne prisutnosti različitih sudionika u raspravi (građanske udruge, itd.). Naposljetku, uočeni su i nedostaci upravne demokracije.

Ključne riječi: uprava, odluke, upravna demokracija 